\title{
Application of Third Generation Synchrotron Source \\ to Studies of Non-Crystalline Materials: In-Se Amorphous Films
}

\author{
A. Burian ${ }^{a, b, *}$, A. JabŁońska ${ }^{a}$, A.M. Burian ${ }^{b}$, \\ D. LeBolloc' ${ }^{c}$, H. Metzger ${ }^{c}$, O. Proux ${ }^{c, d}$, \\ J.L. Hazemann ${ }^{c, d}$, A. Mosset ${ }^{d}$ And D. RaOuX ${ }^{e}$ \\ ${ }^{a}$ A. Chełkowski Institute of Physics, University of Silesia \\ Uniwersytecka 4, 40-007 Katowice, Poland \\ ${ }^{b}$ Centre of Polymer Chemistry, Polish Academy of Sciences \\ M. Skłodowskiej-Curie 34, 41-819 Zabrze, Poland \\ ${ }^{c}$ ESRF, BP 220, 38043 Grenoble, France \\ ${ }^{d}$ Laboratoire de Crystallographie, UPR CNRS 5031 \\ BP 166X, 38042 Grenoble, France \\ ${ }^{e}$ Centre Universitaire Paris-Sud, Bâtiment 209H \\ BP 34, 91898 Orsay Cedex, France
}

\begin{abstract}
The local structure of vacuum evaporated In-Se amorphous films, containing 50, 60, and 66 at.\% Se, was studied using differential anomalous X-ray scattering and extended X-ray absorption fine structure. Both intensity and absorption spectra were measured in the vicinity of the absorption $K$-edge of Se. The differential anomalous X-ray scattering data were converted to real space by the inverse Fourier transform yielding the differential radial distribution functions. The obtained results provide evidence for the presence of Se-In spatial correlations for $\operatorname{In}_{50} \mathrm{Se}_{50}$ and Se-In and Se-Se correlations for $\mathrm{In}_{40} \mathrm{Se}_{60}$ and $\mathrm{In}_{34} \mathrm{Se}_{66}$ within the first coordination sphere.
\end{abstract}

PACS numbers: $61.10 .-\mathrm{i}, 61.43 . \mathrm{Dq}$

${ }^{*}$ corresponding author; e-mail: burian@us.edu.pl 


\section{Introduction}

Binary amorphous semiconducting systems have become of interest recently because of possibilities for applications and contribution to understanding transport and optical properties of non-crystalline materials. Among them compounds of indium and selenium are of importance because of their layered structure in which atoms form strong covalent bonds within a single layer and have weak interlayer interaction of the Van der Waals type. Owing to the layered structure, a low density of dangling bonds is expected which makes it possible to produce heterojunction devices with a low interface density of states [1]. The structural disorder, either configurational or chemical, is the basic factor which determines fundamental properties of amorphous semiconductors, i.e. their electrical and optical characteristics. Configurational disorder refers to fluctuations in both bond lengths and angles. In chemically disordered materials bonding arrangement leads to coordination in which the minority component is surrounded by atoms of both kinds. Coordination defects, dangling bonds and chemical disorder define the energy structure of bands and lead to the occurrence of electronic states deep within the band gap. Therefore precise knowledge of the local environment of selected atomic species is of importance from the point of view of fundamental properties of amorphous semiconductors.

The direct structural information about the structure of amorphous semiconductors comes mainly from wide angle X-ray scattering (WAXS). The conventional WAXS experiment performed on the In-Se amorphous films has shown that the crystalline InSe model, in which each In is tetrahedrally coordinated by 1 In and $3 \mathrm{Se}$ and each Se is bonded to $3 \mathrm{In}$, accounts very well for the experimental data in the range of the interatomic distances up to about $10 \AA$. As the Se content increases the Se-Se correlations occur as the result of statistical occupancy of the In sites by In and Se in proportions depending on the chemical composition [2, 3]. Such conclusions have been drawn from the data for which the inseparable, distinct In-In, In-Se, and Se-Se contributions are merged into the first coordination peak of the radial distribution function (RDF) and therefore more precise structural information is necessary to define unambiguously local surroundings of the constituent atoms. The synchrotron radiation techniques of differential anomalous wide angle X-ray scattering (DAWAXS) and extended X-ray absorption fine structure (EXAFS) offer efficient tools for such a purpose. In the present paper we report on the DAWAXS and EXAFS studies at the Se $K$-edge for the $\operatorname{In}_{50} \mathrm{Se}_{50}$, $\mathrm{In}_{40} \mathrm{Se}_{60}$, and $\mathrm{In}_{34} \mathrm{Se}_{66}$.

\section{Experiments and data analysis}

The amorphous In-Se films were prepared by thermal evaporation of the bulk polycrystalline materials onto glass substrates maintained at room temperature. The preparation technique has been described in detail in our previous papers 
[2-4] and will not be repeated here. The chemical composition of the films was determined by X-ray photoemission spectroscopy. The investigated film compositions were: 50,60 , and 66 at.\% ( \pm 1 at.\%). The Se $K$-edge absorption spectra were recorded at room temperature at the European Synchrotron Radiation Facility (ESRF, Grenoble, France) on the BM32 beam line in a transmission mode in the energy range of 12500-14100 eV. A double-crystal $\mathrm{Si}_{111}$ monochromator and $\mathrm{Ni} / \mathrm{Pt}$-coated mirrors were used with a high harmonic rejection rate and the energy resolution of $\Delta E / E=2 \times 10^{-4}$. The X-ray scattering data were collected on the ID01 beam line at ESRF using incident photon energies tuned exactly at the Se absorption edge $(12653 \mathrm{eV})$ and at $11800 \mathrm{eV}$. In order to calibrate the energy of the experiment the absorption profiles of the investigated samples were measured using the fluorescence method. The energy of the edge was taken at the midpoint of the rise in the absorption edge. The estimated value differs slightly from the tabulated position of the Se $K$-edge $(12658 \mathrm{eV})$ [5]. The observed shift between the experimental and theoretical values is due to the chemical effect, because the theoretical absorption coefficients are calculated for free atoms. A set of a double-crystal $\mathrm{Si}_{111}$ monochromator and mirrors yielded the extremely stable incident beam $( \pm 1 \mathrm{eV})$. A InSb analyzer was used before a detector in order to remove the parasitic Compton and fluorescence intensities. Such an experimental setup allowed to reduce the required correction to minimum. In fact, only the absorption correction was necessary. It is noteworthy that it is important step towards improving a quality of the experimental data when compared with second generation synchrotron sources (see for example [6]).

The EXAFS spectra were analysed using conventional procedures, namely pre- and post-edge background removal, normalization and Fourier filtering with a Kaiser window followed by a curve fitting method. The software package SEDEM was used for all computations [7]. The oscillatory part of the absorption coefficient in the single-scattering approximation can be written as

$$
\chi(k)=\sum_{j} S_{0}^{2} N_{j} F_{j}(k) \exp \left(-2 k^{2} \sigma_{j}^{2}\right) \exp \left(-2 r_{j} / \lambda_{j}\right) \frac{\sin \left[2 k r_{j}+\phi_{i j}(k)\right]}{k r_{j}^{2}},
$$

where $k=\left[8 \pi m\left(E-E_{0}\right) / h^{2}\right]^{1 / 2}, m$ is the mass of electron, $h$ is Planck's constant, $E_{0}$ is the threshold energy, $S_{0}^{2}$ is the amplitude scaling factor included to take into account multielectron excitation at the absorbing atom, $F_{j}$ is the backscattering amplitude from $N_{j}$ neighbouring atoms of the $j$-th type at a distance $r_{j}$ from the absorbing atom $i, \lambda_{j}$ is the electron free path approximated by $\left[\left(3.2 / k^{4}\right)+k\right] / 0.73$ [7], $\sigma_{j}$ is the standard deviation of the interatomic distance and $\phi_{i j}$ is the total phase shift [8]. The backscattering amplitudes and the phase shifts, calculated using the curved-wave approximation according to McKale et al. [9], were taken for all computations in the present work. The value of the scale factor $S_{0}^{2}$ was held fixed at 0.75 during the fitting procedure as given in $[10,11]$. The non-linear Marquardt-Levenberg minimization algorithm was used in the present work to extract the structural parameters in Eq. (1). 
The DAWAXS technique utilizes the abrupt change in the atomic scattering factor of a component near its absorption edge. The difference of the intensities, scattered at two energies $E_{1}=11800 \mathrm{eV}$ and $E_{2}=12653 \mathrm{eV}$ yields the differential structure factor (DSF) $\Delta S\left(K, E_{1}, E_{2}\right)$ :

$$
\Delta S\left(K, E_{1}, E_{2}\right)=\frac{I\left(K, E_{2}\right)-I\left(K, E_{1}\right)}{\left\langle f\left(K, E_{2}\right)\right\rangle^{2}-\left\langle f\left(K, E_{1}\right)\right\rangle^{2}}
$$

and, after Fourier conversion to real space, the differential reduced radial distribution function (DRRDF) $\Delta G(r)[5]$ :

$$
\Delta G(r)=\frac{2}{\pi} \int_{0}^{K_{\max }} K[\Delta S(k)-1] \frac{\sin \left(\pi K / K_{\max }\right)}{\pi K / K_{\max }} \sin (K r) d K
$$

where $\rho_{0}$ indicates the number density, $K=4 \pi \sin \theta / \lambda, 2 \theta$ is the scattering angle and $\lambda$ is the wavelength. The window function in Eq. (2) is used in order to minimize truncation oscillations. $\langle f\rangle=\sum_{i=1}^{2} c_{i} f_{i}$ is the average atomic scattering factor. The differential radial distribution function (DRDF) for a two-component system can be expressed as follows:

$$
\begin{aligned}
& 4 \pi r^{2} \rho_{0} \Delta g(r)=r \sum_{i=1}^{2} \sum_{j=1}^{2} \sum_{k} \frac{c_{i} N_{i j}^{(k)}}{\sqrt{2 \pi} \sigma_{i j}^{(k)} r_{i j}^{(k)}} \\
& \times \int_{0}^{\infty}\left\{\exp \left[-\frac{\left(r^{\prime}-r_{i j}^{(k)}\right)^{2}}{2\left(\sigma_{i j}^{(k)}\right)^{2}}\right]-\exp \left[-\frac{\left(r^{\prime}+r_{i j}^{(k)}\right)^{2}}{2\left(\sigma_{i j}^{(k)}\right)^{2}}\right]\right\} \\
& \times\left[\Delta P_{i j}\left(r-r^{\prime}\right)-\Delta P_{i j}\left(r+r^{\prime}\right)\right] \mathrm{d} r^{\prime}
\end{aligned}
$$

where

$$
\Delta P_{i j}(r)=\frac{1}{\pi} \int_{0}^{\infty} \frac{\Delta W_{i j}}{c} c_{i} \frac{\sin \left(\pi K / K_{\max }\right)}{\pi K / K_{\max }} \cos (K r) \mathrm{d} K
$$

The third sum in Eq. (4) is taken over all the coordination spheres. The differential weighting coefficients are related to the atomic scattering factors $f$ as follows:

$$
\Delta W_{i j}\left(K, E_{1}, E_{2}\right)=c_{i} c_{j} \operatorname{Re}\left[\frac{f_{i}\left(K, E_{2}\right) f_{j}^{*}\left(K, E_{2}\right)-f_{i}\left(K, E_{1}\right) f_{j}^{*}\left(K, E_{1}\right)}{\left\langle f\left(K, E_{2}\right)\right\rangle^{2}-\left\langle f\left(K, E_{1}\right)\right\rangle^{2}}\right]
$$

Equation (4) can be used for determination of the interatomic distances $r_{i j}^{(k)}$, the coordinationn numbers and the standard deviations of the interatomic distances $\sigma_{i j}^{(k)}$ can be obtained using Eq. (4) [5]. The complex atomic scattering factors in the close vicinity of the absorption edge $f=f_{0}+f^{\prime}+\mathrm{i} f^{\prime \prime}$ were determined from the EXAFS data using the Kramers-Kroning relationship as described in [6]. The following values of the real part of the atomic scattering factor $f^{\prime}$ were achieved in the present work: $-9.46,-11.60$ and -10.10 , electrons for $\operatorname{In}_{50} \mathrm{Se}_{50}$, $\mathrm{In}_{40} \mathrm{Se}_{60}$, and $\mathrm{In}_{34} \mathrm{Se}_{66}$, respectively. 


\section{Results and discussion}

The $k^{2}$-weighted filtered EXAFS spectra are shown in Fig. 1. The data were Fourier filtered selecting the first coordination peak of the radial distribution function. It can be seen from inspection of Fig. 1 that the oscillation peaks are shifted towards higher $k$ values for $\mathrm{In}_{40} \mathrm{Se}_{60}$ and $\mathrm{In}_{34} \mathrm{Se}_{66}$ with respect to $\mathrm{In}_{50} \mathrm{Se}_{50}$. The shift is greater for $\mathrm{In}_{34} \mathrm{Se}_{66}$. This observation suggests the presence of a shorter interatomic distance in the films containing 60 and 66 at.\% Se, which can be explained by the occurrence of the Se-Se bonds in the Se-rich films. The Fourier transforms of the experimantal EXAFS data have been shown in [4]. The radial distribution functions exhibited only the first coordination peaks which were split. Increase in the amplitude of the shorter interatomic distance peak and decrease in the longer distance peak for the Se-rich sample were observed. More evident proofs were provided by the curve-fitting method. A one-shell model containing only $\mathrm{Se}-\mathrm{In}$ correlations was considered for $\mathrm{In}_{50} \mathrm{Se}_{50}$, as consequence of previous findings mentioned in Sec. 1. The fitting procedure converged to the values listed in Table. The resulting $k^{2} \chi(k)$ function is compared with the experimental data in Fig. 2 (bottom). The fitting procedure was performed in the $k$ range from 3 to
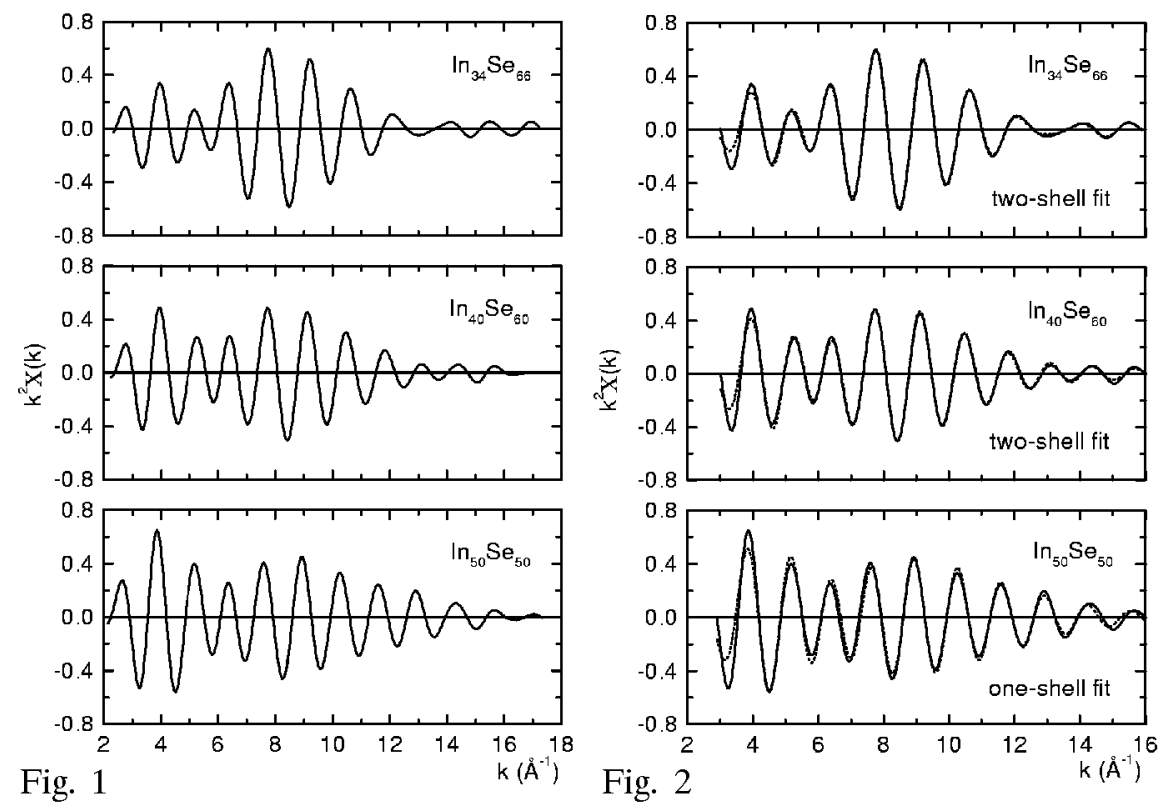

Fig. 1. $k^{2}$-weighted Fourier filtered EXAFS spectra for amorphous $\operatorname{In}_{50} \mathrm{Se}_{50}, \mathrm{In}_{40} \mathrm{Se}_{60}$, and $\operatorname{In}_{34} \mathrm{Se}_{66}$.

Fig. 2. The best-fit model (dotted line) and experimental (solid line) $k^{2} \chi(k)$ functions for amorphous $\mathrm{In}_{50} \mathrm{Se}_{50}, \mathrm{In}_{40} \mathrm{Se}_{60}$, and $\mathrm{In}_{34} \mathrm{Se}_{66}$. 
TABLE

The interatomic distances, the coordination numbers and the $\sigma$ values for amorphous $\operatorname{In}_{50} \mathrm{Se}_{50}, \mathrm{In}_{40} \mathrm{Se}_{60}$ and $\mathrm{In}_{34} \mathrm{Se}_{66}$. The uncertainty estimates are given in the text.

\begin{tabular}{l|c|c|c|c|c|c|c}
\hline \hline Sample & \multirow{2}{*}{ Correlations } & \multicolumn{3}{|c|}{ EXAFS } & \multicolumn{3}{|c}{ DAWAXS } \\
\cline { 3 - 8 } & & $r[\AA]$ & $N$ & $\sigma[\AA]$ & $r[\AA]$ & $N$ & $\sigma[\AA]$ \\
\hline $\mathrm{In}_{50} \mathrm{Se}_{50}$ & $\mathrm{Se}-\mathrm{In}$ & 2.63 & 3.10 & 0.09 & 2.64 & 3.18 & 0.12 \\
$\mathrm{In}_{40} \mathrm{Se}_{60}$ & $\mathrm{Se}-\mathrm{In}$ & 2.60 & 2.60 & 0.09 & 2.58 & 2.10 & 0.20 \\
& $\mathrm{Se}-\mathrm{Se}$ & 2.34 & 0.66 & 0.08 & 2.35 & 0.50 & 0.10 \\
$\mathrm{In}_{34} \mathrm{Se}_{66}$ & $\mathrm{Se}-\mathrm{In}$ & 2.59 & 1.92 & 0.09 & 2.61 & 2.00 & 0.18 \\
& $\mathrm{Se}-\mathrm{Se}$ & 2.35 & 1.20 & 0.07 & 2.34 & 0.60 & 0.18
\end{tabular}

$16 \AA^{-1}$ in order to avoid problems with artefacts coming from Fourier filtering. The calculated and experimental curves are in a very good agreement. For the Se-rich samples a two-shell model in which the Se-In and Se-Se atomic pairs contribute to the first coordination sphere was considered. The resulting structural parameters are shown in Table. An excellent agreement between the models are clearly seen in Fig. 2, providing arguments for validity of the proposed models. The estimated uncertainties of the structural parameters are: $\pm 0.003 \AA, \pm 0.08$ and $\pm 0.01 \AA$ for $r, N$ and $\sigma$, respectively. Square roots of the diagonal elements of the covariance matrix, created during the fitting procedure, were taken as the uncertainties of the structural parameters. However, it is essential to note that such obtained inaccuracies concern only random errors. Systematic errors may lead to increased uncertainty in the obtained results.

The results of the DAWAXS studies are shown in Figs. 3-5. In Fig. 3 the total structure factors (TSF's) and the differential structure factors (DSF's) are presented. The TSF's for $E=11800 \mathrm{eV}$ and $E=12653 \mathrm{eV}$ are shifted by 1 and 1.5 respectively. Both TSF's and DSF's oscillate around the unity line which indicates that the correction and normalization procedures were performed correctly. The total reduced radial distribution functions (TRRDF's) are shown in Fig. 4 together with the DRRDF's. The TRRDF's for $E=11800 \mathrm{eV}$ and $E=12653 \mathrm{eV}$ are shifted by 2 and 4 , respectively. All the curves oscillate around the zero line and do not contain significant oscillations below the first structural peak. Only in the case of the $\operatorname{In}_{50} \mathrm{Se}_{50}$ the spurious peak is observed at $r \approx 0.5 \AA$ in the DRRDF as the result of the rather noisy DSF for this composition. Therefore it can be concluded that the DAWAXS experimental data and the data processing procedures are reliable. The values of the interatomic distances, the coordination numbers and the standard deviations of the interatomic distances, obtained from EXAFS, were taken as the starting parameters for simulations of the DRDF's, performed according to Eq. (4). The resulting parameters are listed in Table. A very good agreement of the simulated and experimental DRDF's, shown in Fig. 5, was at- 

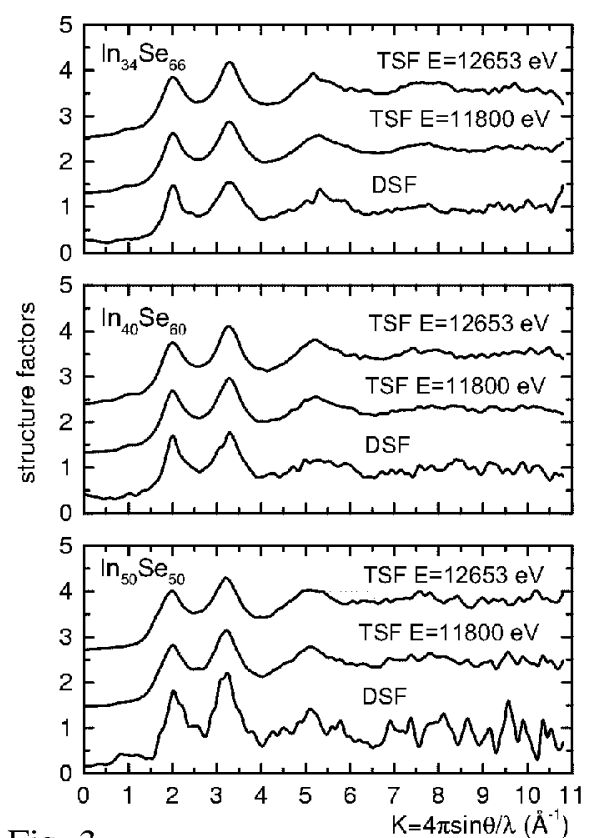

Fig. 3

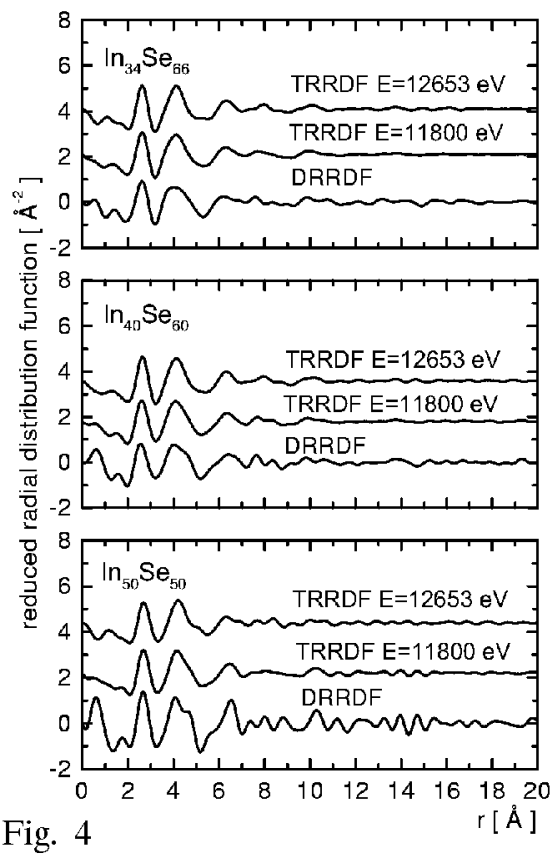

Fig. 4

Fig. 3. Total and differential structure factors for amorphous $\operatorname{In}_{50} \mathrm{Se}_{50}, \mathrm{In}_{40} \mathrm{Se}_{60}$, and $\mathrm{In}_{34} \mathrm{Se}_{66}$. The TSF's for $E=11800 \mathrm{eV}$ and $E=12653 \mathrm{eV}$ are shifted by 1 and 1.5 respectively.

Fig. 4. Total and differential reduced radial distribution function for amorphous $\mathrm{In}_{50} \mathrm{Se}_{50}, \mathrm{In}_{40} \mathrm{Se}_{60}$, and $\mathrm{In}_{34} \mathrm{Se}_{66}$. The TRRDF's for $E=11800 \mathrm{eV}$ and $E=12653 \mathrm{eV}$ are shifted by 2 and 4, respectively.

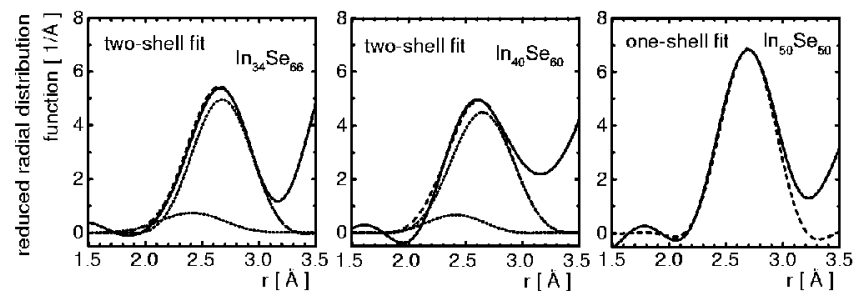

Fig. 5. A comparison of the simulations (dashed line) with experimental differential radial distribution function (solid line) for amorphous $\operatorname{In}_{50} \mathrm{Se}_{50}, \mathrm{In}_{40} \mathrm{Se}_{60}$, and $\mathrm{In}_{34} \mathrm{Se}_{66}$. The partial Se-In and Se-Se components are shown as dotted lines.

tained. The estimated errors are: $\Delta r= \pm 0.02 \AA, \Delta N= \pm 0.25$ and $\Delta \sigma= \pm 0.02 \AA$. The statistical test proposed by Joyner et al. [12] was used to deduce the uncertainty limits on the fitted parameters. The DAWAXS data provide also arguments for the presence of the Se-Se correlations in the $\mathrm{In}_{40} \mathrm{Se}_{60}$ and $\mathrm{In}_{34} \mathrm{Se}_{66}$ films. Comparison of the EXAFS and DAWAXS results shows apparent coincidence between 
them. The interatomic distances are practically the same within the precision of both methods. The DAWAXS coordination numbers are slightly lower than those of EXAFS, nevertheless one can conclude that, on the average, the Se atoms are threefold coordinated. Finally, it is essential to point out that the DAWAXS technique, similarly as EXAFS, is the structural probe of the Se environment, but contains also information about long range ordering.

\section{Conclusions}

The DAWAXS and EXAFS techniques yield a self-consisted and detailed description of the atomic arrangement around Se in the vacuum evaporated amorphous $\mathrm{In}_{50} \mathrm{Se}_{50}, \mathrm{In}_{40} \mathrm{Se}_{60}$, and $\mathrm{In}_{34} \mathrm{Se}_{66}$ films. The application of the third generation synchrotron source offers the possibility of measurements of the pure coherent intensities with statistics good enough to obtain the reliable differential structure factors and the differential reduced radial distribution functions. The use of both experimental methods is especially important when distinct partial correlations are merged in the first coordination sphere.

\section{Acknowledgment}

A.J. would like to thank the State Committee for Scientific Research (Poland) for the research grant No. 5 PO3B 11421.

\section{References}

[1] J.V. McCany, R.B. Murray, J. Phys. C 10, 1211 (1977).

[2] A. Burian, A.M. Burian, J. Weszka, M. Żelechower, P. Lecante, J. Mater. Sci. 35, 3121 (2000).

[3] A. Jabłońska, A. Burian, A.M. Burian, P. Lecante, A. Mosset, J. Alloys Comp. 328, 214 (2001)

[4] A. Jabłońska, A. Burian, A.M. Burian, J. Szade, O. Proux, J.L. Hazemann, A. Mosset, D. Raoux, J. Non-Cryst. Solids, in press.

[5] S. Sasaki, KEK Report 83-229, Nat. Lab. for High Energy Physics, Tsukuba, Japan, 1984.

[6] A. Burian, P. Lecante, A. Mosset, J. Galy, J.M. Tonnerre, D. Raoux, J. Non-Cryst. Solids 212, 23 (1997).

[7] A. Aberdam, J. Synchrotron Rad. 5, 1287 (1998).

[8] B.K. Teo, EXAFS: Basic Principles and Data Analysis, Springer-Verlag, Berlin 1986.

[9] G. McKale, B.W. Veal, A.P. Palinkas, S.K. Chen, G.S. Knapp, J. Am. Chem. Soc. 110, 3763 (1988).

[10] T.A. Carlson, Photoelectron and Auger Spectroscopy, Plenum, New York 1975.

[11] E.A. Stern, B. Bunker, S.M. Heald, in: EXAFS Spectroscopy: Techniques and Applications, Eds. B.K. Teo, D.C. Joy, Plenum, New York 1981, p. 59.

[12] R. Joyner, K.J. Martin, P. Meehan, J. Phys. C 20, 4005 (1987). 